\title{
MONOTONICITY, CONVEXITY AND SYMMETRIC DERIVATES
}

\author{
CLIFFORD E. WEIL
}

\begin{abstract}
If the first lower symmetric derivate of a continuous function is nonnegative, then it is nondecreasing. If the second lower symmetric derivate of a continuous function is nonnegative, then it is convex. In this paper it is shown that if continuity is replaced by Baire one, Darboux in each of these, then the resulting statements are true.
\end{abstract}

1. Introduction. The point of departure for this paper is the following pair of classical theorems.

THEOREM A. If $f$ is continuous on an open interval I and if the first lower symmetric derivate of $f$ is nonnegative on $I$, then $f$ is nondecreasing on $I$.

THEOREM B. If $f$ is continuous on an open interval I and if the second lower symmetric derivate of $f$ is nonnegative on $I$, then $f$ is convex on $I$.

For a proof of Theorem B see for example [3, p. 23]. Actually a stronger theorem is proved there; one where second lower symmetric derivate is replaced by second upper symmetric derivate. Theorem A can be obtained from Theorem B by observing that if $f$ satisfies the conditions of Theorem $A$, then any indefinite integral of $f$ satisfies the conditions of Theorem B. Here it is shown that in both theorems continuity can be replaced by Baire one, Darboux. In addition it is shown that at least in the case of Theorem B continuity cannot be replaced by Darboux alone. Finally it is observed that in the stronger version of Theorem B proved in [3] continuity cannot be replaced by Baire one, Darboux.

2. Preliminaries. In this section the necessary definitions are given as well as the statements of known theorems which will be used in the remainder of the paper. Throughout all functions are real valued.

2.1 Definition. A function defined on an open interval $I$ is of Baire class one if it is the pointwise limit of a sequence of functions each of which is continuous on $I$.

Received by the editors October 29, 1974 and, in revised form, January 3, 1975.

AMS (MOS) subject classifications (1970). Primary 26A21, 26A48, 26A51; Secondary 26A21.

Key words and phrases. First lower symmetric derivate, second lower symmetric derivate, convex, monotone, Baire class one, Darboux functions. 
2.2 Definition. A function defined on an open interval $I$ has the Darboux property (or is Darboux) if the image of every subinterval of $I$ is an interval.

For a discussion of the elementary facts concerning Baire class one functions having the Darboux property see [1].

2.3 Definition. Let $f$ be a function defined on an open interval $I$, and let $x \in I$. For each $h>0$ so small that $x+h$ and $x-h$ both lie in $I$ define

$$
\Delta_{1} f(x, h)=(f(x+h)-f(x-h)) / 2 h
$$

and

$$
\Delta_{2} f(x, h)=(f(x+h)+f(x-h)-2 f(x)) / h^{2}
$$

Also define

$$
L D_{1} f(x)=\lim _{h \rightarrow 0^{+}} \inf _{1} f(x, h)
$$

with $L D_{2} f(x)$ similarly defined.

Next several theorems due to Baire are stated which will be used in the proofs of the results to follow.

2.4 THEOREM. If a function $f$ defined on an interval $I$ is of Baire class one, then for each closed subset $C$ of $I$,

$$
\{x \in C \mid f \text { restricted to } C \text { is continuous at } x\}
$$

is a dense, $G_{\delta}$ subset of $C$.

Recall that a $G_{\delta}$ set is a countable intersection of open sets.

2.5 THEOREM. If $C$ is a closed set, and if for each $n=1,2, \ldots, H_{n}$ is a dense, $G_{\delta}$ subset of $C$, then $H=\bigcap_{n=1}^{\infty} H_{n}$ is also a dense, $G_{\delta}$ subset of $C$.

2.6 THEOREM. If $H$ is $a G_{\delta}$ set and if $H=\bigcup_{n=1}^{\infty} F_{n}$ where each $F_{n}$ is closed relative to $H$, then at least one of the sets $F_{n}$ contains a nonempty set which is open relative to $H$.

The last two theorems are both versions of the Baire Category Theorem.

3. The monotonicity result.

3.1 THEOREM. Suppose $I$ is an open interval and $f$ is a function of Baire class one possessing the Darboux property defined on I. If $L D_{1} f(x) \geqslant 0$ for each $x \in I$, then $f$ is nondecreasing on $I$.

Proof. It suffices to deal with the case $L D_{1} f(x)>0$ for each $x \in I$, for the theorem in this case applied to $f(x)+\epsilon x$ for each $\epsilon>0$ yields the result in the desired case by letting $\epsilon$ tend to zero. Assume in this special case that $f$ is 
not nondecreasing on $I$. Then there are $a_{0}$ and $b_{0}$ in $I, a_{0}<b_{0}$, with $f\left(a_{0}\right)>$ $f\left(b_{0}\right)$. Let $f\left(a_{0}\right)>\alpha>f\left(b_{0}\right)$ and set

$$
E_{\alpha}=\left\{x \in\left[a_{0}, b_{0}\right] \mid f(x) \leqslant \alpha\right\} \text { and } E^{\alpha}=\left\{x \in\left[a_{0}, b_{0}\right] \mid f(x) \geqslant \alpha\right\} .
$$

Suppose that neither $E_{\alpha}$ nor $E^{\alpha}$ contains an interval. Then each subinterval of $\left[a_{0}, b_{0}\right]$ contains both points from $E_{\alpha}$ and points from $E^{\alpha}$. So if $x \in\left[a_{0}, b_{0}\right]$ is a point of continuity of $f$ (there are such points since $f$ is of Baire class one) then $f(x)=\alpha$. Consequently there can be at most one such $\alpha$. Hence assume $\alpha$ is such that $E_{\alpha}$ or $E^{\alpha}$ contains an interval, and suppose for definiteness, it is $E_{\alpha}$. The argument for $E^{\alpha}$ is similar. Let $(a, b) \subset E_{\alpha}$ where

$$
a=\inf \left\{t:(t, b) \subset E_{\alpha}\right\} .
$$

By the Darboux property $f(a) \leqslant \alpha$ and since $f\left(a_{0}\right)>\alpha, a_{0}<a<b \leqslant b_{0}$. So $L D_{1} f(a)>0$. But by choice of $a$, there is a sequence $\left\{h_{n}\right\}$ of positive numbers converging to zero such that $f\left(a-h_{n}\right)>\alpha$. Moreover for $0<h<b-a$, $f(a+h) \leqslant \alpha$. Thus for $n$ sufficiently large

$$
f\left(a+h_{n}\right)-f\left(a-h_{n}\right)<\alpha-\alpha=0
$$

whence $L D_{1} f(a) \leqslant 0$ which is a contradiction.

4. The convexity result. The proof of the convexity theorem is based on the following lemma.

4.1 LEMMA. Let I be an open interval and $f$ a function of Baire class one possessing the Darboux property defined on I with $L D_{2} f(x)>0$ for all $x \in I$. If there is a convex function $g$ defined on I such that $H=\{x \mid f(x)=g(x)\}$ is a dense, $G_{\delta}$ subset of $I$, then $f(x)=g(x)$ for all $x \in I$.

PRoof. First suppose there is an $x \in I$ with $f(x)>g(x)$. Since $H$ is a dense, $G_{\delta}$ subset of $I$, it is possible to choose $a>0$ so small that $\{h>0 \mid x+$ $h \in H\}$ and $\{h>0 \mid x-h \in H\}$ are dense, $G_{\delta}$ subsets of $[0, a]$. By the form of the Baire Category Theorem stated in 2.5,P $=\{h>0 \mid x+h \in H$ and $x-h \in H\}$ is dense in $[0, a]$. Since $g$ is convex on $I$, it is also continuous there. Consequently, by making $a$ smaller if necessary, it may be assumed that $g(x+h)<$ $f(x)$ and $g(x-h)<f(x)$ for all $h \in[0, a]$. Thus, if $h \in P \cap[0, a]$, then $f(x+h)<f(x)$ and $f(x-h)<f(x)$, and therefore $\Delta_{2} f(x, h)<0$. It follows that $L D_{2} f(x) \leqslant 0$ contrary to assumption. Hence, for all $x \in I, f(x) \leqslant g(x)$.

To show that $f(x)=g(x)$ for each $x \in I$ let $J$ and $J^{\prime}$ be any two closed subintervals of $I$ satisfying $J \subset$ int $J^{\prime}$ where int $J^{\prime}$ denotes the interior of $J^{\prime}$, and let $C=J-$ int $H$. Then $C$ is closed and, as is easily seen, the set $K=\{x \in C$ $f(x)<g(x)\}$ is a dense subset of $C$. What is not so easy to see is that $H \cap C$ 
is also a dense subset of $C$. To this end let $x \in C$ and $L$ an open subinterval of $I$ containing $x$. Because $H$ is dense in $I, L \cap J$ contains a point of $H$. If this point is not in int $H$, then it is in $C$. Otherwise it belongs to an interval $L^{\prime}$ of int $H$. Now it is an easy exercise to show that if a continuous function and one with the Darboux property agree on an interval, then they also agree at the endpoints of that interval. Thus the endpoint of $L^{\prime}$ closer to $x$ belongs to $H$ as well as to $L \cap J$ but not to int $H$. Hence, it is in $H \cap L \cap C$. Consequently, $H \cap C$ is a dense, $G_{\delta}$ subset of $C$.

To complete the proof a second dense, $G_{\delta}$ subset of $C$ is needed. Let $x \in$ $K$. Since $f(y)=g(y)$ for $y \in H$ and since $g$ is continuous at $x$,

$$
f(x)<g(x)=\lim _{y \rightarrow x, y \in H} f(y) .
$$

Hence,

$$
\lim _{y \rightarrow x, y \in H}|(f(y)-f(x)) /(y-x)|=\infty .
$$

Therefore, for each positive integer $n$ there is an open subinterval $J_{n, x}$ of $J^{\prime}$ whose midpoint is $x$ and whose length is no more than $2 / n$ such that $|(f(y)-f(x)) /(y-x)|>n$ for each $y \in H \cap J_{n, x}$. Let

$$
G_{n}=\bigcup_{x \in K}\left(J_{n, x}^{\prime} \cap C\right),
$$

where $J_{n, x}^{\prime}$ is the open interval with midpoint $x$ and length half that of $J_{n, x}$. Then $G_{n}$ is an open, dense subset of $C$. The Baire Category Theorem in the form 2.5 then implies that

$$
H^{\prime}=\bigcap_{n=1}^{\infty} G_{n}
$$

is a dense, $G_{\delta}$ subset of $C$ as is $H \cap H^{\prime}$. Let $y \in H \cap H^{\prime}$. Then $f(y)=g(y)$ and for each $n, y \in G_{n}$. Since $g$ is convex on $I$ and since $J^{\prime}$ is a closed subinterval of $I$, there is an $n$ such that for $u$ and $v$ in $J^{\prime}, u \neq v$,

$$
|(g(u)-g(v)) /(u-v)|<n .
$$

Since $L D_{2} f(y)>0$ by choosing an even larger $n$ if necessary it may be assumed that if $0<h<1 / 2 n$, then $\Delta_{2} f(y, h)>0$. Since $y \in G_{n}$, there is an $x \in K$ with $y \in J_{n, x}^{\prime}$. Therefore,

$$
|f(y)-f(x)|>n|y-x|
$$

Because $|y-x|<1 / 2 n, \Delta_{2} f(y,|y-x|)>0$, from which it follows that

$$
f(2 y-x)-f(y)>f(y)-f(x) \text {. }
$$


Since $g(x) \geqslant f(x), g(2 y-x) \geqslant f(2 y-x)$ and $f(y)=g(y), g(2 y-x)-g(y) \geqslant$ $f(2 y-x)-f(y)$ and $f(y)-f(x) \geqslant g(y)-g(x)$. Note that $x, y$, and $2 y-x$ all belong to $J_{n, x}$ and recall that $J_{n, x} \subset J^{\prime}$. Thus, $|g(2 y-x)-g(y)|<n|y-x|$ and $|g(y)-g(x)|<n|y-x|$. Hence,

$$
\begin{aligned}
n|y-x| & >g(2 y-x)-g(y) \geqslant f(2 y-x)-f(y) \\
& >f(y)-f(x) \geqslant g(y)-g(x)>-n|y-x| ;
\end{aligned}
$$

that is

$$
n|y-x|>f(y)-f(x)>-n|y-x|
$$

which contradicts the inequality (*). It must be concluded that $C=\varnothing$; that is, $f(x)=g(x)$ for all $x \in J$. Since $J$ was an arbitrary closed subinterval of $I$ it follows that $f(x)=g(x)$ on $I$.

4.2 REMARK. This lemma is stated in the form in which it will be used to prove the next theorem. The assumption that $f$ be of Baire class one was used nowhere in the proof and could have been dropped from the assumptions. If this assumption is retained the assumption that $H$ is a $G_{\delta}$ set may be omitted and proved from the condition that $f$ is of Baire class one.

Now the Lemma 4.1 is used to prove the second main result.

4.3 THEOREM. Let I be an open interval and $f$ a Baire one, Darboux function defined on I. If $L D_{2} f(x) \geqslant 0$ for each $x \in I$, then $f$ is convex on $I$.

Proof. It suffices to consider the case $L D_{2} f(x)>0$ for each $x \in I$, for the general case can be obtained from this more specific one by adding $e x^{2}$ to $f(x)$ for $\epsilon>0$ and letting $\epsilon$ tend to 0 . Before this assumption can be exploited a certain dense, $G_{\delta}$ set must be constructed. Let

$$
H_{0}=\{x \in \Pi f \text { is continuous at } x\} \cup I^{c},
$$

where $I^{c}$ denotes the complement of $I$. By $2.4, H_{0}$ is a dense, $G_{\delta}$ subset of $R-$ the real line. For each rational number $r$ let $H_{r}=\left\{x \mid x+r \in H_{0}\right\}$. Then clearly each $H_{r}$ is also a dense, $G_{\delta}$ subset of $R$. By the form 2.5 of the Baire Category Theorem

$$
H=\left(\bigcap_{r \in Q} H_{r}\right) \cap I,
$$

where $Q$ is the set of all rational numbers, is a dense, $G_{\delta}$ subset of $I$. Since $H \subset H_{0} \cap I$ every $x \in H$ is a point of continuity of $f$. Moreover it is asserted that if $x \in H, h \in Q$ with $x+h \in I$, then $x+h \in H$ because for each $r \in Q$, $x \in H_{r+h}$; that is $x+r+h \in H_{0}$; or $x+h \in H_{r}$. Thus $x+h \in \bigcap_{r \in Q} H_{r}$, and since $x+h \in I, x+h \in H$. Similarly, if $x \in H, h \in Q$ with $x-h \in I$, then $x-h \in H$. 
Now the assumption that $L D_{2} f(x)>0$ is used. For each $n=1,2,3, \ldots$ let

$$
F_{n}=\left\{x \in H \mid \Delta_{2} f(x, h) \geqslant 1 / n \text { for each } h \in Q, 0<h \leqslant 1 / n\right\} .
$$

Since $L D_{2} f(x)>0$ for each $x \in H$,

$$
\bigcup_{n=1}^{\infty} F_{n}=H .
$$

Next it is shown that each $F_{n}$ is closed in $H$. If $\left\{x_{k}\right\}$ is a sequence of points in $F_{n}$ and $\lim _{k \rightarrow \infty} x_{k}=x_{0} \in H$, and if $h \in Q$ with $0<h \leqslant 1 / n$, then $x_{0}, x_{0}+h$, and $x_{0}-h$ are all points of continuity of $f$. Hence, $\Delta_{2} f(x, h)$ is a continuous function of $x$ at $x_{0}$. Since $x_{k} \in F_{n}$ for each $k, \Delta_{2} f\left(x_{k}, h\right) \geqslant 1 / n$. Therefore, the continuity assertion implies that $\Delta_{2} f\left(x_{0}, h\right) \geqslant 1 / n$. Thus, $x_{0} \in F_{n}$, and therefore, $F_{n}$ is closed in $H$. So by the form 2.6 of the Baire Category Theorem, there is an open interval $J \subset I$ and an integer $n$ such that $H \cap J \subset F_{n}$.

The next goal is to show that $f$ is convex on a subinterval $L$ of $J$. Let $x \in J \cap H$ and let $0<h \leqslant 1 / n$ be a rational number so small that $x-h$ and $x+h$ both lie in $J$. Put $L=[x-h, x+h]$, and let $f_{1}$ be the function defined on $L$ that agrees with $f$ at the endpoints of $L$ and is linear on $L$. Clearly, $f_{1}$ is convex. Let $f_{2}$ be the function defined on $L$ that agrees with $f$ at the endpoints and the midpoint of $L$ and is linear on each of the subintervals $[x-h, x]$, $[x, x+h]$. Since $x \in H \cap J \subset F_{n}$ and since $h$ is a rational number satisfying $0<h \leqslant 1 / n, \Delta_{2} f(x, h) \geqslant 1 / n>0$. Since $h>0$, it follows that

$$
(f(x+h)+f(x-h)-2 f(x)) / h>0
$$

or

$$
(f(x+h)-f(x)) / h>(f(x)-f(x-h)) / h .
$$

That is, the slope of the line segment connecting $(x+h, f(x+h))$ and $(x, f(x))$ is larger than that of the segment connecting $(x, f(x))$ and $(x-h, f(x-h))$. But the graph of $f_{2}$ is composed of these two segments. It can therefore be deduced that, first, $f_{2}$ is convex on $L$ and, second, that $f_{2} \leqslant f_{1}$.

Let $f_{3}$ be the function defined on $L$ that agrees with $f$ at the points $x-h$, $x-h / 2, x, x+h / 2$ and $x+h$, and is linear on the intervals between these points. By the assumptions on $x$ and $h, x+h / 2 \in H \cap J \subset F_{n}$ and hence $\Delta_{2} f(x+h / 2, h / 2)>0$, from which it follows as in the above calculation that the slope of the line segment joining $(x+h, f(x+h))$ and $(x+h / 2, f(x+h / 2))$ is larger than that joining $(x+h / 2, f(x+h / 2))$ and $(x, f(x))$. Likewise since $\Delta_{2} f(x, h / 2)>0$, the slope of the segment joining $(x+h / 2, f(x+h / 2))$ and $(x, f(x))$ is larger than that joining $(x, f(x))$ and $(x-h / 2, f(x-h / 2))$ which in 
turn is larger than the slope of the line segment joining $(x-h / 2, f(x-h / 2))$ and $(x-h, f(x-h))$. Since the graph of $f_{3}$ is made up of the four line segments mentioned above, it can be concluded that $f_{3}$ is convex on $L$ and that $f_{3} \leqslant f_{2}$.

Continue in this fashion to define a decreasing sequence $\left\{f_{k}\right\}$ of functions each convex on $L$, with the property that if $D_{k}$ denotes the set where $f$ and $f_{k}$ agree, then $D_{k} \subset D_{k+1}, k=1,2, \ldots$ and $D=\bigcup_{k=1}^{\infty} D_{k}$ is dense in $L$. It follows that the function

$$
g(x)=\lim _{k \rightarrow \infty} f_{k}(x)
$$

exists on $L$, that $g$ is convex on $L$ and consequently continuous on int $L$, and that $f$ and $g$ agree on $D$. From the last two facts it can be concluded that $f$ and $g$ agree at every point of continuity of $f$ in int $L$; that is, $f$ and $g$ agree on $H_{0} \cap$ int $L$, a dense, $G_{\delta}$ subset of int $L$. Then the preceding lemma implies that $f=g$ on int $L$. Hence, $f$ is convex on int $L$.

What has been proved so far can be summarized by saying that the interval I contains a subinterval on which $f$ is convex. Now this statement applies equally well to any subinterval of $I$. It follows that if $G=\{x \in I \mid$ there is an open interval containing $x$ on which $f$ is convex , then $G$ is an open, dense subset of $I$. To show that $G=I$, let $C=I-G$. Then $C$ is closed, relative to $I$, and hence a $G_{\delta}$ set. Let

$$
H^{\prime}=\{x \in C \mid f \text { restricted to } C \text { is continuous at } x\} .
$$

Then $H^{\prime}$ is a dense, $G_{\delta}$ subset of $C$ and hence a $G_{\delta}$ set. Next for each $x \in C$ let

$$
G_{x}=\{h>0 \mid x+h \in G \text { and } x-h \in G\},
$$

and for each $n=1,2,3, \ldots$ set

$$
F_{n}^{\prime}=\left\{x \in H^{\prime} \mid \Delta_{2} f(x, h) \geqslant 1 / n \text { for } 0<h \leqslant 1 / n, h \in G_{x}\right\} .
$$

Since for each $x, L D_{2} f(x)>0$,

$$
\bigcup_{n=1}^{\infty} F_{n}^{\prime}=H^{\prime}
$$

To verify that each $F_{n}^{\prime}$ is closed in $H^{\prime}$, let $\left\{x_{k}\right\}$ be a sequence of points of $F_{n}^{\prime}$ which converges to $x \in H^{\prime}$, and let $0<h \leqslant 1 / n$ with $h \in G_{x}$. Since $x+h \in G$ and $x-h \in G$, and $f$ is continuous on $G, f\left(x_{k}+h\right) \rightarrow f(x+h)$ and $f\left(x_{k}-h\right)$ $\rightarrow f(x-h)$ as $k \rightarrow \infty$. Since $x \in H^{\prime}$ and $x_{k} \in F_{n}^{\prime} \subset C, f\left(x_{k}\right) \rightarrow f(x)$ as $k \rightarrow$ $\infty$. Therefore, $\Delta_{2} f\left(x_{k}, h\right) \rightarrow \Delta_{2} f(x, h)$ as $k \rightarrow \infty$. Moreover, since $G$ is open, for $k$ sufficiently large $x_{k}+h \in G$ and $x_{k}-h \in G$. Hence, $h \in G_{x_{k}}$ for $k$ large enough, and since $x_{k} \in F_{n}$ and $0<h \leqslant 1 / n, \Delta_{2} f\left(x_{k}, h\right) \geqslant 1 / n$. It follows that 
$\Delta_{2} f(x, h) \geqslant 1 / n$, or $x \in F_{n}^{\prime}$. Thus $F_{n}^{\prime}$ is closed in $H^{\prime}$ for each $n=1,2, \ldots$. Then 2.6 implies that there is an integer $n$ and an open interval $J^{\prime}$ such that $\varnothing \neq J^{\prime} \cap H^{\prime} \subset F_{n}^{\prime}$. Since $f$ has the Darboux property and since $L D_{2} f(x)>0$ for each $x$ in $I$, it can easily be shown that if $f$ is convex on two intervals with a common endpoint, then it is convex on the union of the two intervals. Hence, $C$ is a perfect set and thus $J^{\prime} \cap H^{\prime}$ is infinite. Therefore, it is possible to select a subinterval $J$ of $J^{\prime}$ whose endpoints lie in $C$ and are not endpoints of intervals of $G$ such that $\varnothing \neq J \cap H^{\prime} \subset F_{n}^{\prime}$. It follows that if $J$ intersects a subinterval of $G$ it contains that interval.

Before proceeding with the main line of the proof it is necessary to establish that $f$ is continuous at an endpoint, $x$, of any subinterval, $L$, of $G \cap J$. Properties of convex functions together with the fact that $f$ has the Darboux property imply the continuity of $f$ at $x$ relative to $L$. In addition since $\Delta_{2} f(x, h)$ $>0$ for $h$ sufficiently small, it is easily seen that

$$
\liminf _{y \rightarrow x} f(y) \geqslant f(x) \text {. }
$$

Now for $y \in C \cap J, y$ not the endpoint of an interval of $G \cap J$, since $\Delta_{2} f(y, h)$ $>0$ for $h$ small enough it follows that there is a sequence $\left\{y_{k}^{\prime}\right\}$ of points of $G$ converging to $y$ with $f\left(y_{k}^{\prime}\right)>f(y)$. Since a function convex on an interval attains its maximum at an endpoint of that interval, it may be assumed that $y_{k}^{\prime} \in C$ is an endpoint of an interval of $G \cap J$. Since $F_{n}^{\prime}$ is dense in $C$ and by (1) it follows that there is a sequence $\left\{y_{k}\right\}$ of points of $F_{n}^{\prime}$ converging to $y$ such that

$$
\liminf _{k \rightarrow \infty} f\left(y_{k}\right) \geqslant f(y) \text {. }
$$

The corresponding statement for $y$, any point in $C$, follows from (1). To prove that lim $\sup _{y \rightarrow x} f(y) \leqslant f(x)$ suppose to the contrary that there is a sequence $\left\{y_{k}\right\}$ converging to $x$ and an $\epsilon>0$ with

$$
\liminf _{k \rightarrow \infty} f\left(y_{k}\right) \geqslant f(x)+\epsilon .
$$

First note that $y_{k} \notin L$. If $y_{k} \in G$, then $y_{k}$ may be replaced by a point of $C$ at which $f$ is no smaller. So assume each $y_{k} \in C$. By what was just proved it follows that there is a sequence $\left\{z_{k}\right\}$ of points of $F_{n}^{\prime}$ converging to $x$ with

$$
\liminf _{k \rightarrow \infty} f\left(z_{k}\right) \geqslant f(x)+\epsilon \text {. }
$$

Next let $\left\{x_{k}\right\}$ be a sequence in $L$ converging to $x$. Then $\lim _{k \rightarrow \infty} f\left(x_{k}\right)=f(x)$ as was already noted. Let $w_{k}=2 z_{k}-x_{k}$ so that $x_{k}$ and $w_{k}$ are on opposite sides of $z_{k}$ but equidistant from $z_{k}$. It is possible to select $\left\{x_{k}\right\}$ in such a way that each $w_{k} \in G$. For $k$ so large that $\left|x_{k}-z_{k}\right|<1 / n$, since $\Delta_{2} f\left(z_{k},\left|x_{k}-z_{k}\right|\right)$ $>1 / n>0$, 


$$
\begin{aligned}
\liminf _{k \rightarrow \infty} f\left(w_{k}\right) & \geqslant \liminf _{k \rightarrow \infty} 2 f\left(z_{k}\right)-f\left(x_{k}\right) \\
& \geqslant 2 f(x)+2 \epsilon-f(x)=f(x)+2 \epsilon .
\end{aligned}
$$

As above there is a sequence $\left\{u_{k}\right\}$ of points of $F_{n}^{\prime}$ converging to $x$ with

$$
\liminf _{k \rightarrow \infty} f\left(u_{k}\right) \geqslant f(x)+2 \epsilon .
$$

Repeating the above procedure it is possible to construct for each $m$ a sequence $\left\{u_{m, k}\right\}$ of points in $F_{n}^{\prime}$ converging to $x$ with

$$
\liminf _{k \rightarrow \infty} f\left(u_{m, k}\right) \geqslant f(x)+m \epsilon .
$$

Finally by a diagonal method pick a sequence $\left\{v_{k}\right\}$ of points of $F_{n}^{\prime}$ converging to $x$ with

$$
\liminf _{k \rightarrow \infty} f\left(v_{k}\right)=\infty
$$

To obtain the contradiction let $y \in G, y \notin L$ with

$$
|x-y|<\min [1 / n, \text { length of } L]
$$

and let $x_{k}=2 v_{k}-y$. Then for $k$ sufficiently large, $x_{k} \in L$. But since $\left|v_{k}-y\right|$ $<|x-y|<1 / n$ and since $y$ and $x_{k}$ are both in $G$,

$$
f\left(x_{k}\right)>2 f\left(v_{k}\right)-f(y),
$$

so that

$$
\liminf _{k \rightarrow \infty} f\left(x_{k}\right)=\infty
$$

contrary to

$$
\lim _{k \rightarrow \infty} f\left(x_{k}\right)=f(2 x-y)
$$

Let $(a, b)$ and $(c, d)$ be two disjoint subintervals of $G \cap J$ with $b<c$, and let $F$ be the function defined on $[a, d]$ by setting $F=f$ on $[a, b]$ and on $[c, d]$ and by letting $F$ be linear on $[b, c]$. It will be shown that $F$ is convex on $[a, d]$. Let $\delta_{1}>0$ be such that if $0<h<\delta_{1}$, then $\Delta_{2} f(b, h)>0$. Let $a<a^{\prime}<b$ with $b-a^{\prime}<\delta_{1}$. It suffices to show that $(c, f(c))$ lies above the line through the points $\left(a^{\prime}, f\left(a^{\prime}\right)\right)$ and $(b, f(b))$, for if this statement is true for every such $a^{\prime}$, then it can easily be seen that $F$ is convex on $[a, c]$. Applying this result to $f(-x)$ yields that $F$ is convex on $[b, d]$. Since the intervals $[a, c]$ and $[b, d]$ overlap in an interval of positive length, it follows that $F$ is convex on $[a, d]$.

To make the proof easier assume that $f\left(a^{\prime}\right)=f(b)=0$. This can be done 
by subtracting a linear function from $f$ which does not change any of the assumptions on $f$. (For a proof that the sum of a Baire one, Darboux function and a continuous function is Baire one, Darboux, see [1, Theorem 7.5, p. 109]. That $L D_{2} f(x)>0$ still holds is trivial.) Let

$$
\gamma=\sup _{y \in[b, c]} f(y)
$$

and let

$$
x=\inf \left\{y \in[b, c] \mid \gamma=\limsup _{z \rightarrow y} f(z)\right\} .
$$

(That the above set is nonempty is easily verified.) Clearly $\gamma=\lim _{\sup _{z \rightarrow x}} f(z)$. Since $f$ is convex on $[a, b]$ and since $f\left(a^{\prime}\right)=f(b)=0, f(b-h) \leqslant 0$ for each $0<h \leqslant b-a^{\prime}$. In addition, since $b-a^{\prime}<\delta_{1}, \Delta_{2} f(b, h)>0$. Thus $f(b+h)>$ $-f(b-h) \geqslant 0$, whence, $f(y)>0$ on $\left[b, b+\left(b-a^{\prime}\right)\right]$. Therefore, $\gamma>0$. The object is to show that $x=c$. First, since $f$ is continuous at $b$ and since $f(b)=$ $0, x \neq b$.

Suppose, contrary to desire, that $b<x<c$. It will be shown next that in this case $x$ is not a point of continuity of $f$ which means that $x \notin G$ nor is $x$ the endpoint of a maximal interval of $G$. For if $f$ is continuous at $x$, then $f(x)=\gamma$. If $h>0$ is such that $x+h \in[b, c]$, and $x-h \in[b, c]$, then $f(x+h) \leqslant \gamma$ and $f(x-h) \leqslant \gamma$, and hence $\Delta_{2} f(x, h) \leqslant 0$, contradicting the assumption $L D_{2} f(x)$ $>0$.

Let $(\alpha, \beta)$ be a subinterval of $G,(\alpha, \beta) \subset(x, x+1 / 2 n) \cap[b, c]$. (Recall $H^{\prime} \cap J \subset F_{n}^{\prime}$.) For each $y \in(\alpha, \beta), f(y) \leqslant \gamma$. By the result of the above paragraph there is $0<\delta_{2}<(\beta-\alpha) / 4$ such that $x+\delta_{2} \in C$ and $x+\delta_{2}<\alpha$. Then

$$
\delta_{2}<\alpha-x<\beta-x<1 / 2 n \text {, and } 4 \delta_{2}<\beta-\alpha \text { or } 2 \delta_{2}-\beta<-2 \delta_{2}-\alpha \text {. }
$$

Hence, $2 x+2 \delta_{2}-\beta<2 x-2 \delta_{2}-\alpha$. Let

$$
L=\left(2 x+2 \delta_{2}-\beta, 2 x-2 \delta_{2}-\alpha\right) .
$$

Since $x-\left(2 x-2 \delta_{2}-\alpha\right)=\alpha-x+2 \delta_{2}>3 \delta_{2}>0, L$ is an interval to the left of $x$ whose significance will be seen once a certain element $z$ of $H^{\prime}$ is selected. Let $\epsilon>0$. There is a $z \in[b, c]$ close to $x$ such that $f(z)>\gamma-\epsilon / 2$. Using an argument similar to one used in the proof that $f$ is continuous at endpoints of intervals of $G \cap J$, it may be assumed that $z \in H^{\prime}$, and $|z-x|<\delta_{2}$. Choose $h$ so that $z-h \in L$. Then

$$
2 x+2 \delta_{2}-\beta<z-h<2 x-2 \delta_{2}-\alpha,
$$

or multiplying by -1 and adding $2 z$ 


$$
-2 x+2 \delta_{2}+\alpha+2 z<z+h<-2 x-2 \delta_{2}+\beta+2 z .
$$

But $-\delta_{2}<z-x<\delta_{2}$. So $0<2 z-2 x+2 \delta_{2}$ and $-2 x+2 z-2 \delta_{2}<0$. Therefore, $\alpha<z+h<\beta$. That is, if $z-h \in L$, then $z+h \in(\alpha, \beta) \subset G$. Since $|z-x|<\delta_{2}$ and since $z+h \in(\alpha, \beta), h>0$. Moreover,

$$
h=x-z+z+h-x<\delta_{2}+\beta-x<1 / 2 n+1 / 2 n=1 / n .
$$

So if, in addition, $z-h \in L \cap G$ (and there are such numbers $h$ since $G$ is dense), then $\Delta_{2} f(z, h)>0$ because $z \in H^{\prime} \subset F_{n}^{\prime}$. So

$$
f(z-h)>2 f(z)-f(z+h)>2(\gamma-\epsilon / 2)-\gamma=\gamma-\epsilon .
$$

That is, for $y \in L \cap G, f(y)>\gamma-\epsilon$. This inequality holds for every $\epsilon>0$. Hence for $y \in L \cap G, f(y) \geqslant \gamma$. This contradicts the choice of $x$. Therefore, $x=c$ as desired. Since $f$ is continuous at $c$ it follows that $f(c)=\gamma>0$ which completes the proof that $F$ is convex on $[a, d]$.

To finish the proof of the theorem let $\left(a_{1}, b_{1}\right)$ be a maximal interval of $G$ contained in $J$, and let $\left(a_{2}, b_{2}\right)$ be a second such interval but to the right of $\left(a_{1}, b_{1}\right)$. Order the remaining maximal intervals of $G$ in $\left(b_{1}, a_{2}\right)$ in a sequence $\left\{\left(a_{k}, b_{k}\right)\right\}_{k=3}^{\infty}$, and note that the interval $\left[b_{1}, a_{2}\right]$ contains points of $C$. Let $f_{1}$ be the function that agrees with $f$ on $\left[a_{1}, b_{1}\right]$ and on $\left[a_{2}, b_{2}\right]$ and is linear on the interval $\left[b_{1}, a_{2}\right]$. Let $f_{2}$ be the function that agrees with $f$ on the intervals $\left[a_{1}, b_{1}\right],\left[a_{2}, b_{2}\right]$, and $\left[a_{3}, b_{3}\right]$ and is linear on the intervals $\left[b_{1}, a_{3}\right]$ and $\left[b_{3}, a_{2}\right]$. This function is convex on $\left[a_{1}, b_{3}\right]$ and on $\left[a_{3}, b_{2}\right]$. Since these two intervals overlap in $\left[a_{3}, b_{3}\right]$, an interval with positive length, it follows that $f_{2}$ is convex on $\left[a_{1}, b_{2}\right]$. Since $f_{1}$ is linear on $\left[b_{1}, a_{2}\right]$, and since $f_{2}$ is convex there, $f_{2}(x) \leqslant f_{1}(x)$ for $x \in\left[b_{1}, a_{2}\right]$. Elsewhere on $\left[a_{1}, b_{2}\right], f_{1}$ and $f_{2}$ agree. Thus $f_{2} \leqslant f_{1}$. In this fashion construct a decreasing sequence $\left\{f_{k}\right\}$ of convex functions such that $f_{k}$ agrees with $f$ on $\left[a_{1}, b_{1}\right],\left[a_{2}, b_{2}\right], \ldots$, and $\left[a_{k+1}, b_{k+1}\right]$. Let $g(x)=\lim _{k \rightarrow \infty} f_{k}(x)$. Then $g$ is convex and agrees with $f$ on $G \cap\left[a_{1}, b_{2}\right]$. Since $G \cap\left[a_{1}, b_{2}\right]$ is an open, dense subset of $\left[a_{1}, b_{2}\right]$, Lemma 4.1 implies that $f$ is convex on $\left[a_{1}, b_{2}\right]$. Hence $\left(a_{1}, b_{2}\right) \subset G$ contrary to the above observation that $\left[b_{1}, a_{2}\right]$ contains points of $C$. Therefore $C=\varnothing$, and hence $G=I$ and consequently $f$ is convex on $I$.

5. Examples. This paper is concluded with two examples which give some indication of the extent to which the two main results can be strengthened. The first shows that in Theorem 4.3 the assumption that the function be of Baire class one cannot be omitted. The second shows that in the same theorem the second lower symmetric derivate cannot be replaced by the second upper symmetric derivate. 
5.1 EXAMPLE. There is a (nonmeasurable) function $f$ that takes on every value in every interval and that satisfies the equation $f(x+y)=f(x)+f(y)$.

REMARK. That the function attains every value in every interval implies that it has the Darboux property. Because of the additivity condition

$$
f(x+h)+f(x-h)-2 f(x)=0 .
$$

Thus, $L D_{2} f(x)=0$, but $f$ is not convex.

Proof. The example is based on the existence of a Hamel basis, $B$, for the real numbers as a vector space over the rational numbers. Write

$$
B=\bigcup_{n=1}^{\infty} B_{n}
$$

where the sets $B_{n}, n=1,2, \ldots$, are uncountable and pairwise disjoint. By multiplying each element of $B_{n}$ by an appropriate rational number, it may be assumed that

$$
B_{n} \subset(1-1 / n, 1+1 / n), \quad n=1,2, \ldots
$$

For each $n=1,2, \ldots$ let $f$ map $B_{n}$ onto $R$ and then extend $f$ from $B$ to all of $R$ by linearity. The equation

$$
f(x+y)=f(x)+f(y)
$$

is then trivial (in fact does not depend on how $f$ is defined on $B$ ). To show that $f$ attains every value in every interval, let $(a, b) \subset R$ and $y \in R$. Pick a nonzero rational number $r \in(a, b)$. Since multiplication by $r$ is a continuous function, there is a positive integer $n$ such that if $x \in(1-1 / n, 1+1 / n)$, then $r x \in(a, b)$. By the definition of $f$, there is an $h \in B_{n}$ such that $f(h)=y / r$. Since $h \in B_{n} \subset$ $(1-1 / n, 1+1 / n), r h \in(a, b)$ and $f(r h)=r f(h)=y$.

5.2 ExAMPle. There is a Baire one, Darboux function whose upper second symmetric derivate is nonnegative which is not convex.

Proof. In [2] Croft proves the existence of a Baire one, Darboux function that is nonnegative, zero almost everywhere, but not identically zero. Let $f$ be the negative of the Croft example. Then $f$ is Baire one, Darboux. For each $x, f(x+h)=f(x-h)=0$ for almost every $h>0$. Hence the upper second symmetric derivate of $f$ at $x$ is

$$
\lim _{h \rightarrow 0^{+}}-2 f(x) / h^{2} \geqslant 0
$$

since $f(x) \leqslant 0$. That $f$ is not convex seems clear.

6. Acknowledgements. The author would like to thank Professor Richard J. O'Malley for suggesting the present form of the proof of the monotonicity 
theorem which is much simpler than the original and also Professor C. J. Neugebauer whose careful refereeing produced a significant shortening of the proof of the convexity theorem.

\section{BIBLIOGRAPHY}

1. A. M. Bruckner and J. G. Ceder, Darboux continuity, Jber. Deutsch. Math.-Verein. 67 (1964/65), Abt. 1, 93-117. MR 32 \#4217.

2. H. Croft, $A$ note on a Darboux continuous function, J. London Math. Soc. 38 (1963), 9-10. MR 26 \#5103.

3. A. Zygmund, Trigonometric series. Vol. I, Cambridge Univ. Press, New York, 1959. MR 21 \#6498.

DEPARTMENT OF MATHEMATICS, MICHIGAN STATE UNIVERSITY, EAST LANSING, MICHIGAN 48823 